\title{
Commentary
}

\section{Heparin: A drug of varying composition and effectiveness}

\author{
William W. Coon, M.D. Ann Arbor, Mich. \\ The Department of Surgery, Section of General Surgery, University of \\ Michigan Medical Center
}

In the past few years concerns have been expressed by clinical investigators regarding the variable and unpredictable risk of both hemorrhage and thrombosis in patients receiving heparin. Recent observations tend to indicate that a number of elements may contribute to variability in response to heparin. Possible factors which may be responsible include differences in chemical composition and in methods of assay, variable absorption, different types and degrees of alteration in coagulation mechanism of patients, and inaccuracies or inadequacies in methods for monitoring therapy.

Several commercial heparins have been shown to contain 21 components varying in molecular weight ${ }^{12}$ and in elemental composition, electrophoretic mobility, and nuclear magnetic spectrum. ${ }^{8}$ When several assay methods are compared, a 12-fold difference in activity has been found between heparin of relatively low molecular weight and higher molecular weight material. ${ }^{1}$ If preparations of commercial heparin are isolated on matrix-bound antithrombin III, a large proportion of the material

\footnotetext{
Reprint requests to: Dr. William W. Coon, Department of Surgery, Section of General Surgery, University of Michigan Medi-
} cal Center, 1405 E. Ann, Ann Arbor, Mich. 48109. has been shown to have low activity while the remaining "active" one third contains $85 \%$ of the anticoagulant effect. ${ }^{7}$

The problems of reproducibility of the in vitro coagulation tests used for assay of heparin as defined in the United States Pharmacopeia and British Pharmacopaeia have been known for many years, but no more satisfactory method has yet been developed. It is not surprising that in vitro clotting tests performed on animal plasma may not reflect the antithrombotic effectiveness of heparin in vivo in human whole blood. While standardization of preparations of heparin in vivo in man or animals would be more expensive, it might also prove to be considerably more reliable ${ }^{10}$; a problem with utilization of in vivo assay in animals is the differences in hemostatic mechanisms between species. Ideally, if heparin standards *were homogeneous and could be characterized by physical and chemical methods, a chemical procedure might be developed, but this would require the acquisition of considerably more information than is now available on the relationship between physicochemical differences in heparin preparations and antithrombotic effect. 
Variations in assays of heparin activity have resulted when a preparation from one tissue or species is compared with a standard from another tissue or species. ${ }^{3}$ However, this finding together with the known heterogeneity of tissue heparins cannot be extrapolated to imply that currently available commercial heparins, when administered in a fixed dose in United States Pharmacopeia units, vary in anticoagulant activity with species or tissue source. In the past few years poorly documented claims that beef lung heparin is superior to porcine mucosal heparin have been published. These statements have not been substantiated when carefully controlled double-blind crossover trials measuring in vivo anticoagulant effect have been conducted. ${ }^{2,} 11$

Differences in frequency of bleeding have been reported when several preparations of heparin are injected subcutaneously. Since the calcium salt of heparin was associated with a lower prevalence of hemorrhagic problems than the sodium salt, it was initially thought to be a preferable product. A recent study, however, has shown that subcutaneous injection of calcium heparin results in lower blood levels of heparin than those achieved by injection of the same number of units of sodium heparin, indicating that a lesser amount of the former is absorbed. ${ }^{15}$ The molecular weight of heparin also influences degree of subcutaneous absorption. ${ }^{9}$ Although clinicians have recognized for some time that the anticoagulant response after subcutaneous injection may be highly variable, particularly with relatively small doses, these are the first good data to indicate that the structure of the heparin molecule may influence absorption. Binding of heparin to tissue proteins or inactivation by tissue enzymes may also be involved, but this has not yet been documented.

There is considerable evidence that, in some instances, "resistance" to the antithrombotic effects of heparin may be related to the altered physiology of the patient rather than to the drug itself. Patients with very extensive thrombotic processes, especially in conjunction with malignant neoplasm or certain septicemias or endotoxemias, and those with a vasculitis are very refractory to heparin therapy. Certain subjects with recurrent venous thrombosis have been reported to have more satisfactory therapeutic responses from antiplatelet agents than from prolonged heparin therapy. The rare patients with antithrombin III deficiency and recurrent thromboses do not have an adequate anticoagulant response to heparin because the principal action of heparin is mediated by an acceleration of the complexing of antithrombin with thrombin and other serine proteases. The efficacy of low doses of subcutaneous heparin in the prevention of venous thromboembolism varies with the nature of the patient's primary problem, being of limited benefit in subjects with hip fracture or total hip replacement, presumably because the extensive trauma to tissues accompanying these conditions brings about a coagulative abnormality or results in a degree of venous trauma which is less responsive to the antithrombotic effects of low doses of heparin. Although the above explanation appears to explain the failure of heparin therapy in these conditions, it remains speculative and does not eliminate the possibility that an improved and uniformly potent heparin preparation might be more effective in treatment.

An equally important element in the variable effectiveness of heparin may be the result of clinical errors in the method of administration and in dose and procedures for monitoring therapy. Recent pharmacokinetic studies have shown that after intravenous injection heparin is distributed in the plasma volume ${ }^{5}$ and has a mean anticoagulant half-life in the circulation of $90 \mathrm{~min}$. These findings would favor the calculation of dose on the basis of ideal body weight (as a reflection of plasma volume) and the administration of drug either by continuous infusion or very frequent intermittent intravenous injection. Although this approach is being increasingly adopted, there is at present no uniform method of therapy. A recent randomized prospective trial has shown that patients receiving constant infusions of heparin had an approximately 8-fold reduction in hemorrhagic complications when compared with a group receiving intermittent intravenous injections; there were too few thrombotic complications to determine differences. ${ }^{14}$ The decreased frequency of bleeding is readily explainable since the excessive prolongation of clotting tests produced 
by an intravenous bolus of heparin is avoided. An important ancillary finding of the above study was that, with the use of a predetermined level of anticoagulation as reflected by the activated partial thromboplastin time (APTT), the patients receiving constant infusion required about $25 \%$ less heparin than those receiving bolus injections. On the basis of this and another similar prospective trial, ${ }^{6}$ continuous infusion appears to lessen the risk of hemorrhage over that observed in patients receiving intermittent intravenous injections at 4-hr intervals and also to lessen the dosage of drug. Hemorrhagic risk from intermittent injections might also be reduced if Estes' ${ }^{5}$ recommendation for injection at $1 \frac{1}{2}-\mathrm{hr}$ intervals is followed, but this routine would place an excessive burden upon nursing staff. Subcutaneous injection of heparin at 8-or 12-hr intervals may be equally effective in reduction of frequency of bleeding complications, but such a prospective trial has not yet been conducted. None of these routes of administration has been shown to be superior as far as reduction of thrombotic sequelae in man is concerned.

A major issue in improvement of the effectiveness of heparin therapy is whether periodic monitoring of the level of anticoagulation is of value. Ideally, if one knew the level of anticoagulation which was associated with maximum antithrombotic effectiveness and minimum risk of bleeding, there would be no question; but these two criteria may be incompatible since optimal suppression of thrombosis may require the acceptance of an appreciable incidence of bleeding. In addition, current measures of "anticoagulant" activity may not be the most suitable procedures to define antithrombotic effect.

Although monitoring patients receiving intermittent intravenous injections of heparin has not reduced risk of hemorrhage, ${ }^{14}$ clotting tests have been performed during constant infusions or periodic subcutaneous injections of heparin in order to assure an adequate anticoagulant effect and prevent excessive prolongation of clotting in the face of considerable interindividual variability in response. Basu and associates ${ }^{4}$ have reported that the 5 patients who developed recurrent thromboembolism in a group of sub- jects receiving constant infusions of equivalent amounts of heparin for treatment of venous thromboembolism had significantly lesser prolongations of APTT than patients without recurrence. Although this observation supports the premise that anticoagulant and antithrombotic effect are correlated, conflicting opinions have been expressed concerning the adequacy of the APTT for monitoring heparin therapy.

A principal objection to this test is that an appreciable proportion of hospitalized patients not receiving heparin have abnormal prolongations of the APTT. In addition, the standard procedure usually requires modification and careful calibration if it is used to determine blood levels of heparin; certain reagents (e.g., ellagic acid) are unsuitable since they make the test relatively insensitive to varying concentrations of heparin.

Many investigators have advocated other procedures, such as thrombin time, assay of factor $\mathrm{Xa}$ inactivation, polybrene titration, or activated recalcification times, as being more accurate or more sensitive to heparin effects. For the most part, Lee-White clotting times have been abandoned as too time consuming and inaccurate. As Rosenberg ${ }^{13}$ has recently pointed out, however, all of these procedures are based upon the determination of the degree of "activation" by heparin of plasma antithrombin by measuring in vitro its effect upon an added amount of a serine protease or its inhibitory effect upon serine proteases generated in plasma in vitro by kaolin addition (APTT). He questions whether these techniques reflect the effect of heparinactivated antithrombin on serine proteases circulating in vivo and proposes that a more satisfactory monitoring technique might be based upon measurement in vivo of levels of the several serine proteases (factors XII, XI, X, IX, ii). If such an approach could be developed and if it proves to be practical and economically feasible, it might provide a mechanism for "finetuning" of the coagulation mechanism so that lesser amounts of heparin would be required.

Some of the factors which have been discussed as possibly responsible for the variability in effectiveness of heparin may not be correctable. It seems quite likely, however, that in view of the recent demonstration of "active" 
and "inactive" forms of heparin, the composition of this drug can be made more uniform. Greater knowledge concerning the physical and chemical structure of the molecule could lead to development of a chemical assay which is more accurate than current methods. Since individual patients will always differ in their coagulation profile, further clinical investigation of the therapeutic effectiveness of differing methods of administration and techniques for monitoring level of anticoagulation and antithrombotic efficacy may be productive in lessening hemorrhagic and thrombotic complications. Some of the variability in response to heparin in certain patients may be related to a concomitant deficiency of antithrombin; administration of exogenous antithrombin in conjunction with heparin might improve its antithrombotic effect.

\section{References}

1. Andersson, L. O., Barrowcliffe, T. W., Holmes, E., Johnson, E. A., Sims, G. E. C.: Anticoagulant properties of heparin fractionated by affinity chromatography on matrix-bound antithrombin III and by gel filtration, Thromb. Res. 9:575-583, 1976.

2. Baltes, B. J., Diamond, S. and D'Agostino, R. J.: Comparison of anticoagulant activity of two preparations of purified heparin, Clin. PHARMACOL. THER. 14:287-290, 1973.

3. Bangham, D. R., and Woodward, P. M.: A collaborative study of heparins from different sources, Bull. W.H.O. 42:129-149, 1970.

4. Basu, D., Gallus, A., Hirsh, J., and Cade, J.: A prospective study of the value of monitoring heparin treatment with activated partial thromboplastin time, N. Engl. J. Med. 287:324-327, 1972.

5. Estes, J. W.: The fate of heparin in the body, Curr. Ther. Res. 18:45-57, 1975.
6. Glazier, R. L., and Crowell, E. B.: Randomized prospective trial of continuous versus intermittent heparin therapy, J. A. M. A. 236:13651367, 1976.

7. Höök, M., Björk, I., Hopwood, J., and Lindahl, U.: Anticoagulant activity of heparin: Separation of high-activity and low-activity heparin species by affinity chromotography on immobilized antithrombin, FEBS Lett. 66:90-93, 1976.

8. Jaques, L. B., and Kavanaugh, L. W.: Variability of heparin preparations in clinical use, Thromb. Haemostas. 56:(Suppl.)171-180, 1973.

9. Johnson, E. H., Kirkwood, T. B. L., Stirling, Y., Perez-Requijo, J. L., Ingram, G. I. C., Bangham, D. R., Brozovic, M.: Four heparin preparations: Anti-Xa potentiating effect of heparin after subcutaneous injection, Thromb. Haemostas. 35:586-591, 1976.

10. Kuo, S. H., Jaques, L. B., and Millar, G. I.: An improved in vivo procedure for standardization of heparin preparations, J. Pharm. Pharmacol. 24:858-868, 1972.

11. McMahon, F. G., Jain, A. K., Ryan, J. R., and Lefton, T. E.: Anticoagulant potency of mucosal and lung heparin, Clin. Pharmacol. Ther. 17:79-82, 1975.

12. Nader, H. B., McDuffie, N. M., and Dietrich, C. P.: Heparin fractionation by electrofocusing: Presence of 21 components of different molecular weights, Biochem. Biophys. Res. Commun. 57:488-493, 1974.

13. Rosenberg, R. D.: Heparin action, Circulation 49:603-605, 1974.

14. Salzman, E. W., Deykin, D., Shapiro, R. M., and Rosenberg, R. D.: Management of heparin therapy; controlled prospective trial, N. Engl. J. Med. 292:1042-1050, 1975.

15. Thomas, D. P., Sagar, S., Stomatakis, J. D., Maffei, F. H. A., Erdi, A., Kakkar, V. V.: Plasma heparin levels after administration of calcium and sodium salts of heparin, Thromb. Res. 9:241-245, 1976. 